\title{
Alelo doble mutante en el gen EXT1 no informado previamente en una adolescente con exostosis múltiple hereditaria
}

\author{
Double mutant alleles in the EXT1 gene not previously reported in a teenager \\ with hereditary multiple exostoses
}

\author{
Dr. Francisco Cammarata-Scalisi, ${ }^{a}$ Téc. Mónica Cozar, ${ }^{b}$ Dr. Daniel Grinberg, ${ }^{b}$ Dra. Susana Balcells, ${ }^{b}$ \\ Dra. Carla G. Asteggiano, Lic. E. Gustavo Martínez-Domenech, ${ }^{c}$ Dra. Ana Bracho, ${ }^{d}$ Dra. Yanira Sánchez, ${ }^{d}$ \\ Dra. Frances Stock, ${ }^{e}$ Dr. Wilmer Delgado-Luengo, ${ }^{d}$ Dra. Carmen Zara-Chirinos ${ }^{d}$ y Dr. José Antonio Chacín ${ }^{d}$
}

\begin{abstract}
RESUMEN
Las formas hereditarias de exostosis múltiple, actualmente denominada EXT1/EXT2-CDG dentro de los desórdenes congénitos de la glicosilación, son los tumores óseos benignos más comunes y se caracterizan por la formación de lesiones óseas cubiertas de cartílago, localizadas en yuxtaposición a epífisis de huesos largos, aunque, en los casos graves, pueden presentar una amplia distribución. El inicio es variable desde los 2-3 años hasta los 13-15 y presenta una incidencia estimada que va de $1 / 18000$ a 1/50000 casos en los países europeos. Se presenta el caso de un doble alelo mutante en el gen EXT1 no informado previamente en una adolescente y su familia con exostosis múltiple hereditaria.

Palabras clave: exostosis múltiple hereditaria, EXT1/EXT2-CDG.
\end{abstract}

\section{ABSTRACT}

Hereditary forms of multiple exostoses, now called EXT1/ EXT2-CDG within Congenital Disorders of Glycosylation, are the most common benign bone tumors in humans and clinical description consists of the formation of several cartilagecapped bone tumors, usually benign and localized in the juxta-epiphyseal region of long bones, although wide body dissemination in severe cases is not uncommon. Onset of the

a. Unidad de Genética Médica. Departamento de Puericultura y Pediatría. Facultad de Medicina. Universidad de Los Andes. Mérida, Venezuela.

b. Departamento de Genética. Facultad de Biología. Universidad de Barcelona. CIBERER, IBUB. Barcelona, España.

c. CONICET-CEMECO. Centro de Estudio de las Metabolopatías Congénitas (UNC). Departamento de Farmacología. Facultad de Medicina. Universidad Católica de Córdoba. Córdoba, Argentina.

d. Instituto de Investigaciones Genéticas. Facultad de Medicina. Universidad del Zulia. Maracaibo, Venezuela.

e. Unidad de Oncología Pediátrica. Instituto Autónomo Hospital Universitario de Los Andes. Mérida, Venezuela.

Correspondencia:

Dr. Francisco Cammarata-Scalisi:

francocammarata19@gmail.com

Conflicto de intereses: ninguno que declarar.

Recibido: 29-8-2014

Aceptado: 7-11-2014 disease is variable ranging from 2-3 years up to 13-15 years with an estimated incidence ranging from $1 / 18000$ to $1 / 50000$ cases in European countries. We present a double mutant alleles in the EXT1 gene not previously reported in a teenager and her family with hereditary multiple exostoses.

Key words: hereditary multiple exostoses, EXT1/EXT2-CDG.

http:/ /dx.doi.org/10.5546/aap.2015.e109

\section{INTRODUCCIÓN}

Las formas hereditarias de exostosis múltiple (EMH) u osteocondromatosis múltiple, actualmente denominadas EXT1/ EXT2-CDG dentro de los desórdenes congénitos de la glicosilación, son entidades de herencia autosómica dominante debidas a defectos en la formación de O-glicoconjugados y caracterizadas por la formación de osteocondromas, que son los tumores óseos benignos más comunes. Se caracterizan por la formación de lesiones óseas cubiertas de cartílago, localizadas en yuxtaposición a epífisis de huesos largos, aunque, en los casos graves, pueden presentar una amplia distribución. Dependiendo de su número, tamaño y ubicación, pueden causar complicaciones, como deformidad con limitación funcional, compresión de estructuras adyacentes e, incluso, la transformación a condrosarcoma, que puede ocurrir en el 3-5\% de los casos. ${ }^{1-4}$

El inicio es variable, desde los 2-3 años hasta los 13-15. Presentan una incidencia estimada que va de $1 / 18000$ a $1 / 50000$ casos en los países europeos. ${ }^{5}$ Son causadas principalmente por mutaciones en genes supresores de tumores, como el EXT1, localizado en 8q24.11, que causa la EMH tipo 1 (OMIM 133700); el EXT2, ubicado en 11p11.2, que causa la EMH tipo 2 (OMIM 133701); y la EMH tipo 3 (OMIM 600209), cuyo locus ha sido localizado en el brazo corto del cromosoma 19 (19p), pero aún no se han detectado alteraciones de este. ${ }^{1,6,7}$ Las mutaciones en EXT1 representan entre 56 y $78 \%$ 
de todos los casos de EMH y, en EXT2, entre 21 y $44 \%$. Ambos genes codifican las enzimas glicosiltransferasas localizadas en retículo endoplásmico, $\mathrm{N}$-acetilglucosamina transferasa y ácido D-glucorónico transferasa, implicadas en la síntesis de las cadenas del heparán sulfato (HS) y proteoglucanos de heparán sulfato, el cual interviene en el mecanismo de señalización en la placa de crecimiento del hueso endocondral y cuyas alteraciones conducirían a la formación de osteocondroma. ${ }^{1,6}$

Se presenta el caso de una adolescente evaluada de forma multidisciplinaria con diagnóstico clínico, estudio radiológico y molecular de EMH con doble alelo mutante en el gen EXT1 no informado previamente.

\section{CASO CLÍNICO}

Adolescente femenina de 12 años y 7 meses, referida para su evaluación por presentar prominencias óseas en extremidades desde el año de edad.

Padre de 39 años y madre de 38 años, no consanguíneos. El padre y otros integrantes de su familia presentan un fenotipo similar (Figura 1).

Producto de V gesta, obtenida por cesárea segmentaria a las 38 semanas. El peso y la talla al nacer fueron $3100 \mathrm{~g}$ y $49 \mathrm{~cm}$.

Evidenció adecuado desarrollo psicomotor y presentó buen desenvolvimiento escolar.

Fue intervenida en tres ocasiones por exéresis de lesiones; a los cuatro años, en ambos antebrazos y en el pie derecho; a los ocho años, en el fémur derecho; y a los diez años, en el húmero izquierdo y radio y cúbito ipsilateral.

Examen físico: talla de $138 \mathrm{~cm}$ (P 10-3); peso de $31 \mathrm{~kg}$ (P 10-3); circunferencia cefálica de 54 cm (P 50-75). Normocefalia con región frontal

FIGURA 1. Genealogía en la que se muestra trasmisión de las formas hereditarias de exostosis múltiple en cuatro generaciones y un patrón de herencia autosómico dominante

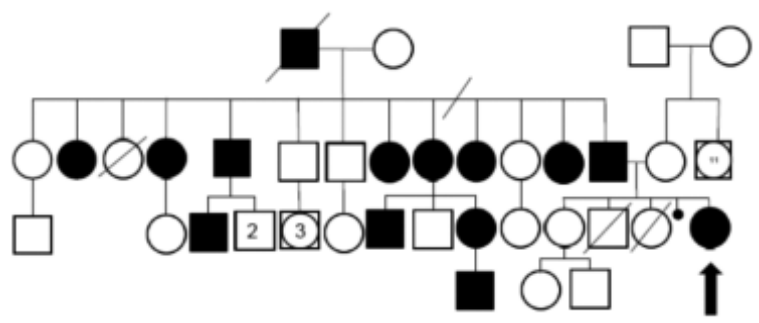

amplia. Puente nasal en silla de montar con punta bulbosa. El paladar era ojival, con malposición y maloclusión dentaria y piezas dentales con cambios en la coloración. En las extremidades, se evidenció acortamiento de miembro superior izquierdo, con limitación en la extensión del codo, genu valgum e inserción proximal del $4^{\circ}$ ortejo derecho.

Estudios de radiografía simple de antebrazos evidenciaron distorsión de la arquitectura con lesiones encondromatosas a nivel de la región epifisiaria y metafisiaria distal de radio y cúbito a predominio izquierdo (Figura 2) y en tercio distal de fémur bilateral.

Mediante la técnica de amplificación por reacción en cadena de la polimerasa (PCR), seguida de secuenciación automática, se detectaron las mutaciones en heterocigosis c. $1284+1 \mathrm{G}>\mathrm{A}$ en el intrón 4 y la c. $1184 \mathrm{C}>\mathrm{G}$ (p.Ser395Cys) en el exón 4 del gen EXT1, además de la c.710C $>$ T (p.Ser237Leu) igualmente en heterocigosis, en el exón 6 del gen EXT2.

La mutación encontrada en una de las dos copias del gen EXT1 altera el empalme del exón 4 del gen; situada en el sitio dador de empalme del intrón 4, es un cambio de guanina por adenina. Esta posición es $100 \%$ conservada, por lo que su modificación debe considerarse patogénica. Se trata, además, de una mutación no descrita en la base de datos: "Multiple Osteochondroma Mutation Database" (http://medgen.ua.ac.be/LOVDv2.0/home. php) ni en $\mathrm{HGMD}^{\circledR}$ Professional 2013, 4-15 de diciembre de 2013. En la misma copia del gen EXT1 y en el mismo exón 4, se ha identificado otra mutación, que provoca la sustitución del aminoácido serina por cisteína, en la posición 395 de la exostosina 1, e igualmente se trata de una mutación no descrita en las dos bases de datos ya mencionadas. En la segunda copia de EXT1, no se encontró ninguna mutación.

Además, en una copia del gen EXT2, se ha encontrado un cambio de citosina a timina en la

FIGURA 2. Radiografía de antebrazo, en la que se evidencian osteocondromas en la región epifisiaria y metafisiaria distal de radio y cúbito izquierdo

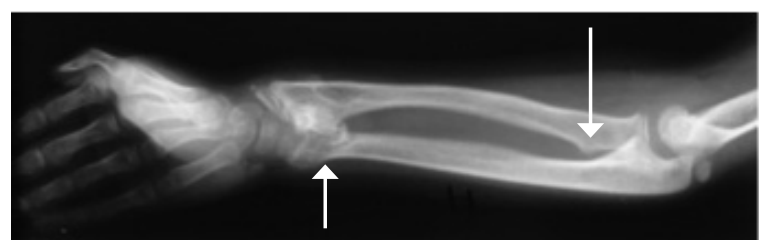


posición 710 del exón 6, que produce un cambio de aminoácido serina por leucina en la posición 237 de la exostosina 2. Esta mutación ha sido documentada por Wuyts, et al. ${ }^{8}$ que la consideran de patogenecidad dudosa, pues la presenta un paciente y también su padre no afecto.

\section{DISCUSIÓN}

La EMH se caracteriza por una amplia variabilidad clínica intrafamiliar e interfamiliar. ${ }^{3}$ La limitación de la movilidad articular se encuentra entre las complicaciones más frecuentes. La estatura puede verse afectada en $37 \%$ en el sexo masculino y $44 \%$ en el femenino. Dado que la mayoría de los casos son asintomáticos al momento del nacimiento, el diagnóstico precoz solo se puede realizar por cribado en las familias con antecedentes de la entidad. La articulación de la rodilla está afectada en $94 \%$ de los casos y, en las EMH graves, se pueden producir escoliosis ${ }^{9}$ o compresión de la médula espinal. ${ }^{10}$

La paciente descrita presenta solo lesiones en huesos largos de las extremidades y, según la clasificación mostrada en la Tabla 1,3 representa el tipo III A, debido a la limitación en la extensión del codo en el miembro superior izquierdo. La evaluación médica debe ser multidisciplinaria, en forma individual, seguir la evolución natural y la aparición de posibles complicaciones.

Las dos mutaciones en heterocigosis encontradas en el gen EXT1 son consideradas patogénicas y no fueron documentadas en las bases de datos consultadas. Además, en el gen EXT2 de este caso, se consiguió una mutación ya conocida, pero de patogenicidad dudosa. ${ }^{8}$ Las mutaciones encontradas en el gen EXT1 fueron

TABla 1. Clasificación clínica de formas hereditarias de exostosis múltiple

\begin{tabular}{|c|c|}
\hline Clase & Hallazgos \\
\hline $\begin{array}{ll}\text { I } & \\
\text { I A } \\
\text { I B }\end{array}$ & $\begin{array}{l}\text { Sin deformidad y limitación funcional } \\
\quad \leq 5 \text { sitios con exostosis } \\
>5 \text { sitios con exostosis }\end{array}$ \\
\hline $\begin{array}{l}\text { II } \\
\text { II A } \\
\text { II B }\end{array}$ & $\begin{array}{l}\text { Con deformidad y sin limitación funcional } \\
\qquad \begin{array}{l}\quad 5 \text { sitios con deformidad } \\
>5 \text { sitios con deformidad }\end{array}\end{array}$ \\
\hline $\begin{array}{l}\text { III } \\
\text { III A } \\
\text { III B }\end{array}$ & $\begin{array}{l}\text { Con deformidad y limitación funcional } \\
\text { Limitación funcional en un sitio } \\
\text { Limitación funcional en más de un sitio }\end{array}$ \\
\hline
\end{tabular}

igualmente encontradas en una tía paterna afectada, pero esta no presentó la mutación en el gen EXT2. Estos análisis se realizaron en el Departamento de Genética, de la Facultad de Biología de la Universidad de Barcelona, en España. En dicha institución, estudiaron a 39 pacientes españoles, 37 de ellos con mutaciones, $29(74,4 \%)$ en el gen EXT1 y $8(20,5 \%)$ en EXT2, 18 de estas no documentadas. Con el objeto de establecer correlación genotipo-fenotipo, se evidenció un menor número de exostosis en mutaciones con cambio de sentido. ${ }^{11}$

En el Instituto Ortopédico de Rizzoli, en Boloña, Italia, se estudiaron 529 pacientes. De estos, $344(65 \%)$ presentaron mutaciones en el gen EXT1 y, 134 (25,3\%), en EXT2. Las pacientes de sexo femenino, las mutaciones en el gen EXT2 y la ausencia de mutaciones en EXT1/2 se asociaron con un fenotipo leve. En contraste, un fenotipo grave se asoció con el sexo masculino y las mutaciones en EXT1. La transformación maligna se observó en 26 (5\%) de los pacientes, pero no se encontró asociación en la aparición de condrosarcoma y las variables anteriormente comentadas. $^{3}$

En Argentina, en el Centro de Estudio de las Metabolopatías Congénitas (CEMECO), se desarrolla un programa latinoamericano para la detección de esta entidad y, hasta el momento, fueron detectados los alelos mutantes en 78\% de los pacientes con EMH. Por PCR y MLPA (Multiplex ligation-dependent probe amplification), se detectaron 21 mutaciones patogénicas: 15 en el gen EXT1 y 6 en EXT2. De estas, 10 mutaciones fueron no descritas previamente. Los datos clínicos en 21 (78\%) de los pacientes demostraron un fenotipo moderado en $15 \%$ y presentación grave en $63 \%$. Dos pacientes desarrollaron transformación maligna a condrosarcoma en pelvis, que ocasionó una compresión vascular y de órganos cercanos muy grave. ${ }^{4}$

Entre los principales diagnósticos diferenciales, se encuentran la enfermedad de Ollier, entidad de etiología incierta, con una incidencia de 1/100000, la cual se caracteriza por múltiples encondromas con marcada predominancia unilateral y riesgo de transformación maligna, que se puede incrementar a 35\%. Además, el síndrome de Maffucci, igualmente de etiología incierta, es más infrecuente y se caracteriza por la asociación de encondromas con hemangiomas. Tanto la enfermedad de Ollier como el síndrome de Maffucci pueden presentar lesiones en diferentes regiones, con especial predilección en manos. ${ }^{7}$ 
Se presentó un caso de EMH con diagnóstico clínico, radiológico y molecular, y se encontraron dos mutaciones patogénicas no informadas previamente en el gen EXT1 y otra considerada dudosa en el gen EXT2. Este hallazgo molecular muestra mutaciones que hacen importante el seguimiento clínico, ante la posibilidad de asociar estas mutaciones a una eventual característica de presentación de la entidad. Además, se debe brindar un oportuno asesoramiento genético, ya que los integrantes de la familia que presentan la alteración tienen un riesgo de $50 \%$ de trasmitir la entidad a sus descendientes.

\section{REFERENCIAS}

1. Ciavarella M, Coco M, Baorda F, Stanziale P, et al. 20 novel point mutations and one large deletion in EXT1 and EXT2 genes: report of diagnostic screening in a large Italian cohort of patients affected by hereditary multiple exostosis. Gene 2013;515(2):339-48.

2. Kok HK, Fitzgerald L, Campbell N, Lyburn ID, et al. Multimodality imaging features of hereditary multiple exostoses. Br J Radiol 2013;86(1030):20130398.

3. PedriniE, Jennes I, Tremosini M, Milanesi A, etal. Genotypephenotype correlation study in 529 patients with multiple hereditary exostoses: identification of "protective" and "risk" factors. J Bone Joint Surg Am 2011;93(24):2294-302.

4. Delgado MA, Sarrión P, Azar N, Zecchini L, et al. A novel nonsense mutation of the EXT1 gene in an Argentinian patient with multiple hereditary exostoses: a case report. J Bone Joint Surg Am 2012;94(11):e76.

5. De Andrea CE, Hogendoorn PC. Epiphyseal growth plate and secondary peripheral chondrosarcoma: the neighbours matter. J Pathol 2012;226(2):219-28.

6. Jamsheer A, Socha M, Sowi ska-Seidler A, Telega K, et al. Mutational screening of EXT1 and EXT2 genes in Polish patients with hereditary multiple exostoses. J Appl Genet 2014;55(2):183-8.

7. Cammarata-Scalisi F, Sánchez-Flores R, Stock-Leyton F, Labrador-Chacón N, et al. Exostosis múltiple hereditaria. Reporte de un caso y diagnóstico diferencial de las encondromatosis. Acta Ortop Mex 2012;26(6):388-92.

8. Wuyts W, Radersma R, Storm K, Vits L. An optimized DHPLC protocol for molecular testing of the EXT1 and EXT2 genes in hereditary multiple osteochondromas. Clin Genet 2005;68(6):542-7.

9. RyckxA,Somers JF,AllaertL. Hereditary multipleexostosis. Acta Orthop Belg 2013;79(6):597-607.

10. Ashraf A, Larson AN, Ferski G, Mielke CH, et al. Spinal stenosis frequent in children with multiple hereditary exostoses. J Child Orthop 2013;7(3):183-94.

11. Sarrión P, Sangorrin A, Urreizti R, Delgado A, et al. Mutations in the EXT1 and EXT2 genes in Spanish patients with multiple osteochondromas. Sci Rep 2013;3:1346. 